\title{
Equações e programa computacional para cálculo do transporte de solutos do solo
}

\author{
João C. F. Borges Júnior ${ }^{1} \&$ Paulo A. Ferreira ${ }^{2}$
}

\begin{abstract}
RESUMO
Em função deste trabalho, objetivou-se desenvolver e testar um programa computacional para calcular os parâmetros das equações de transporte de solutos no solo, com base no ajustamento de modelos teóricos a dados observados, e executar simulações para a variação espacial e temporal da concentração e do balanço de massa de solutos no perfil do solo. Utilizou-se o método dos mínimos quadrados (Levenberg-Marquardt) para obtenção dos estimadores dos parâmetros coeficiente dispersivo-difusivo e fator de retardamento. O programa desenvolvido, denominado Disp, possui interface gráfica que torna simples o seu uso quanto aos procedimentos de entrada de dados, execução dos cálculos e acesso aos resultados. Nos formulários de resultados, gráficos e tabelas relacionados às curvas de efluente podem ser gerados além da possibilidade de se executar simulações quanto à variação espacial e temporal da concentração e do balanço de massa de solutos no perfil do solo. Testes comparativos entre o Disp e o programa CXTFIT, relativos aos cálculos dos parâmetros número de Peclet e fator de retardamento, indicaram equivalência entre os dois programas, porém a interface gráfica do Disp o torna de uso mais simples em relação ao CXTFIT.
\end{abstract}

Palavras-chave: deslocamento miscível, fator de retardamento, coeficiente dispersivo-difusivo

\section{Equations and computer program for calculating the solute transport in soil}

\begin{abstract}
This study aimed to develop and to test a computer program for calculating the parameters of soil solute transport equations, based on adjustment of theoretical models to observed data, as well as to perform simulations for the space and temporary variations of the concentration and balance of the solute mass in the soil profile. The least-squares method (LevenbergMarquardt) was used to obtain the estimators of the diffusion-dispersion coefficient and retardation factor parameters. The developed program, so-called DISP, is provided with a graphic interface that makes possible its use in procedures for data input, accomplishment of calculations and access to results. In the result forms, a number of graphs and tables relative to the effluent curves can be generated besides the possibility to accomplish simulations concerning to space and time variations of both concentration and balance of the solute mass in the soil profile. Comparative tests between DISP and the program CXTFIT, relative to the calculations of the Peclet number and retardation factor parameters, pointed out equivalence between both programs, however the graphic interface in DISP makes its use more feasible in relation to CXTFIT.
\end{abstract}

Key words: miscible displacement, retardation factor, diffusion-dispersion coefficient 


\section{INTRODUÇÃO}

O deslocamento de fluidos miscíveis é um processo que ocorre quando um fluido se mistura a outro e o desloca no perfil do solo. A lixiviação de sais no solo, devido a chuvas ou irrigações, é um exemplo de deslocamento miscível; outro exemplo é o processo de mistura e deslocamento envolvendo uma solução aplicada, via irrigação, e a solução do solo.

Geralmente, a agricultura moderna usa fertilizantes e pesticidas benéficos à cultura e que não causam problemas ambientais mais sérios quando retidos na superfície do solo e na região radicular, desde que sejam observados critérios racionais de aplicação. O deslocamento desses insumos, para partes mais profundas do perfil, ameaça a qualidade da água freática, além de torná-los indisponíveis às culturas; portanto, a adequação na aplicação de insumos agrícolas contribui para a preservação do meio ambiente, além de otimizar o perfil financeiro do empreendimento, pois permite maiores produtividades e minimiza o desperdício.

Quando um fluido é deslocado por um outro fluido em uma coluna de solo, a interface entre o fluido deslocador e o deslocado perde nitidez ou definição a medida em que o tempo transcorre, em razão da mistura entre eles. Esta mistura decorre da difusão de solutos de um fluido para outro e da dispersão, em virtude da diferença de velocidade de escoamento dentro de um mesmo poro e de poros de diferentes dimensões. Às vezes, um soluto é aplicado no solo e, em seguida, é aplicada água pura para transportá-lo no perfil do solo. Este procedimento, comum em fertirrigação, constitui um pulso, ou seja, aplicam-se alguns milímetros de água pura seguidos de água contendo fertilizantes dissolvidos e, finalmente, a água pura (Ferreira, 2001).

A preocupação com o destino de determinados produtos químicos aplicados no solo tem motivado vários pesquisadores a desenvolverem modelos teóricos, objetivando descrever os processos físicos envolvidos no transporte desses produtos no perfil do solo. Por outro lado, o advento desses modelos despertou, nos pesquisadores, um outro problema relativo à quantificação adequada dos parâmetros envolvidos nas equações de transporte de solutos no solo, como o fator de retardamento e o coeficiente dispersivo-difusivo.

Tem sido consenso entre os pesquisadores que o método mais adequado para estimar esses parâmetros é o ajustamento dos modelos teóricos a dados experimentais de laboratório e campo, empregando-se programas computacionais, como o programa CXTFIT (Toride et al., 1999), o qual requer, nas versões livres, um laborioso preparo dos arquivos de entrada, não sendo também de fácil comunicação com o usuário.

O desenvolvimento de programas computacionais desta natureza traz ganhos inerentes à modelagem, como detecção de demandas de conhecimento e de dados, discernimento entre hipóteses alternativas e acesso à metodologia implementada, o que possibilita alterações e aperfeiçoamentos, quando necessários, no modelo.

Com este trabalho, teve-se como objetivo primordial desenvolver e testar um programa computacional para o cálculo dos parâmetros das equações de transporte de solutos no solo, com base no ajustamento de modelos teóricos a dados observados, e para executar simulações quanto à variação espacial e temporal da concentração e do balanço de massa de soluto no perfil do solo, no decorrer de processos de deslocamento de fluidos miscíveis.

\section{MATERIAL E MÉTODOS}

Os métodos mais eficazes, para se estimar os parâmetros das equações diferenciais de transporte de solutos no solo, são baseados no ajustamento de soluções analíticas dessas equações diferenciais a dados experimentais, empregandose programas computacionais.

Como as soluções matemáticas são resultados da submissão das equações diferenciais a determinadas condições iniciais e de contorno, o usuário, ao preferir uma delas, deve adequar o seu modelo físico, colunas em laboratório ou experimento no campo, àquelas condições sob as quais a solução foi obtida. Os resultados serão equivocados se, por exemplo, o usuário colher dados de concentração no efluente e usar uma solução para concentração residente.

\section{Equação de transporte - concentração residente}

Ao se aplicar determinada solução numa coluna de solo previamente lixiviado com água pura, a interface entre a solução e a água perde nitidez a medida que avança no perfil; isto ocorre em razão dos efeitos combinados da difusão do soluto e da dispersão hidrodinâmica que, por sua vez, é conseqüente à geometria irregular do meio poroso e ao atrito de cisalhamento, inerente ao escoamento dentro de cada poro.

A seguinte equação diferencial descreve o transporte de soluto no solo em uma dimensão (van Genuchten \& Wierenga, 1986; Reichardt, 1996)

$$
\frac{\partial}{\partial t}\left(\theta C_{r}+\rho S\right)=-\frac{\partial}{\partial x}\left(\theta D \frac{\partial C_{r}}{\partial x}-q C_{r}\right)
$$

em que

$$
\begin{aligned}
& \mathrm{t} \text { - tempo, } \mathrm{T} \\
& \theta \text { - teor de água do solo, em base volume, } \mathrm{L}^{3} \mathrm{~L}^{-3} \\
& \mathrm{C}_{\mathrm{r}} \text { - concentração residente ou concentração na so- } \\
& \text { lução do solo, } \mathrm{M} \mathrm{L}^{-3} \\
& \rho \text { - massa específica do solo, } \mathrm{M} \mathrm{L}^{-3} \\
& \mathrm{~S} \text { - concentração adsorvida (massa de soluto por } \\
& \text { unidade de massa de solo) } \\
& \mathrm{X} \text { - distância em relação a superfície do solo, L } \\
& \mathrm{D} \text { - coeficiente dispersivo-difusivo, } \mathrm{L}^{2} \mathrm{~T}^{-1} \\
& \mathrm{q} \text { - densidade de fluxo, } \mathrm{L} \mathrm{T}^{-1}
\end{aligned}
$$

Na Eq. 1 assume-se que o soluto não está sujeito a processos de produção ou decaimento. Segundo van Genuchten \& Wierenga (1986), coeficientes relativos à produção e decaimento podem ser requeridos em estimativas de transporte de certos produtos orgânicos e formas de nitrogênio.

Conforme van Genuchten \& Wierenga (1986) e Ferreira (2001), considera-se uma relação linear entre $S$ e $C_{r}$, numa condição isotérmica, de forma 


$$
\mathrm{S}=\mathrm{kC}_{\mathrm{r}}
$$

em que k é um coeficiente empírico de distribuição, $\mathrm{L}^{3} \mathrm{M}^{-1}$.

Considerando-se, também, que o escoamento é permanente em um meio homogêneo ( $\theta$ e q constantes no tempo e no espaço), a Eq. 1 reduz-se a

$$
\mathrm{R} \frac{\partial \mathrm{C}_{\mathrm{r}}}{\partial \mathrm{t}}=\mathrm{D} \frac{\partial^{2} \mathrm{C}_{\mathrm{r}}}{\partial \mathrm{x}^{2}}-\mathrm{v} \frac{\partial \mathrm{C}_{\mathrm{r}}}{\partial \mathrm{x}}
$$

em que v é a velocidade de escoamento $\left(\mathrm{L} \mathrm{T}^{-1}\right)$ e $\mathrm{R}$ (adimensional) é o fator de retardamento, expresso por

$$
\mathrm{R}=1+\frac{\rho \mathrm{k}}{\theta}
$$

Quando não ocorrer reação entre o soluto e o solo, k será igual a 0 e, conseqüentemente, $\mathrm{R}$ igual a 1 .

Condições iniciais e de contorno - concentração residente $\left(C_{r}\right)$

A solução particular da Eq. 3 deve vir acompanhada de equações auxiliares, descrevendo as condições iniciais e de contorno do sistema a ser estudado.

A condição inicial é considerada como

$$
\mathrm{C}_{\mathrm{r}}(\mathrm{x}, 0)=\mathrm{C}_{\mathrm{i}}
$$

em que $C_{i}$ é a concentração inicial do soluto em apreço, $\mathrm{M} \mathrm{L}^{-3}$.

Conforme Parker \& van Genuchten (1984), será utilizada, na seção de entrada $(\mathrm{x}=0)$, uma condição de contorno tipo 3 ou tipo fluxo, recomendável para a aplicação de um pulso, isto é:

$$
\left.\left(\mathrm{C}_{\mathrm{r}}-\frac{\mathrm{D}}{\mathrm{V}} \frac{\partial \mathrm{C}_{\mathrm{r}}}{\partial \mathrm{x}}\right)\right|_{\mathrm{x}=0}= \begin{cases}\mathrm{C}_{0} & 0<\mathrm{t} \leq \mathrm{t}_{0} \\ 0 & \mathrm{t}>\mathrm{t}_{0}\end{cases}
$$

em que:

$$
\begin{gathered}
\mathrm{C}_{0} \text { - concentração da solução aplicada, constante, } \\
\quad \mathrm{M} \mathrm{L}^{-3} \\
\mathrm{t}_{0} \text { - tempo de aplicação da solução, } \mathrm{T}
\end{gathered}
$$

A Eq. 6 indica descontinuidade na concentração, evidenciada pelas condições de contorno de entrada, a qual aumenta com o valor da dispersividade aparente, igual a D/v. Esta descontinuidade é conseqüência direta da suposição de que, no plano de injeção, existe estrato de espessura infinitesimal no qual os parâmetros do sistema mudam descontinuamente, desde aqueles de um reservatório com mistura perfeita $(\mathrm{x}<0)$ até aqueles do meio poroso $(x>0)$. Microscopicamente, esta mudança sempre ocorre numa região finita de transição (Parker \& van Genuchten, 1984).

Conforme van Genuchten \& Wierenga (1986), para um sistema semi-infinito a condição de contorno quando $\mathrm{x} \rightarrow \infty$ é escrita como

$$
\frac{\partial \mathrm{C}_{\mathrm{r}}}{\partial \mathrm{x}}(\infty, \mathrm{t})=0
$$

\section{Solução para $\mathrm{C}_{\mathrm{r}}$ - Modelo 1}

Segundo Parker \& van Genuchten (1984), a solução para a Eq. 3, sujeita às equações 5 a 7 , é

$$
C_{r}(x, t)=\left\{\begin{array}{lc}
C_{i}+\left(C_{0}-C_{i}\right) A_{2}(x, t) & 0<t \leq t_{0} \\
C_{i}+\left(C_{0}-C_{i}\right) A_{2}(x, t)-C_{0} A_{2}\left(x, t-t_{0}\right) & t>t_{0}
\end{array}\right.
$$

em que:

$$
\begin{aligned}
A_{2}(x, t) & =\frac{1}{2} \operatorname{erfc}\left[\frac{R x-v t}{2(D R t)^{1 / 2}}\right]+\left(\frac{v^{2} t}{\pi D R}\right)^{1 / 2} \exp \left[-\frac{(R x-v t)^{2}}{4 D R t}\right] \\
& -\frac{1}{2}\left(1+\frac{v x}{D}+\frac{v^{2} t}{D R}\right) \exp \left(\frac{v x}{D}\right) \operatorname{erfc}\left[\frac{R x+v t}{2(D R t)^{1 / 2}}\right]
\end{aligned}
$$

em que:

$$
\begin{aligned}
& \text { erfc - função erro complementar } \\
& \exp \text { - função exponencial }
\end{aligned}
$$

Os números adimensionais de Peclet, $\mathrm{P}$, e de volume de poros, Vp, percolados de uma coluna de solo de comprimento L, são calculados por meio das expressões

$$
\begin{gathered}
P=\frac{v L}{D} \\
V p=\frac{v t}{L}
\end{gathered}
$$

As equações 12 e 13, obtidas a partir das equações 8 e 9, para x igual a L, mostram a solução para $C_{r}$ em termos de Vp e P.

$\mathrm{C}_{\mathrm{r}}(\mathrm{Vp})=\left\{\begin{array}{lc}\mathrm{C}_{\mathrm{i}}+\left(\mathrm{C}_{0}-\mathrm{C}_{\mathrm{i}}\right) \mathrm{A}_{2}(\mathrm{Vp}) & 0<\mathrm{Vp} \leq \mathrm{Vp}_{0} \\ \mathrm{C}_{\mathrm{i}}+\left(\mathrm{C}_{0}-\mathrm{C}_{\mathrm{i}}\right) \mathrm{A}_{2}(\mathrm{Vp})-\mathrm{C}_{0} \mathrm{~A}_{2}\left(\mathrm{Vp}-\mathrm{Vp}_{0}\right) & \mathrm{Vp}>\mathrm{Vp}_{0}\end{array}\right.$

em que:

$$
\begin{aligned}
\mathrm{A}_{2}(\mathrm{Vp}) & =\frac{1}{2} \operatorname{erfc}\left[\left(\frac{\mathrm{P}}{4 \mathrm{RVp}}\right)^{1 / 2}(\mathrm{R}-\mathrm{Vp})\right]+\left(\frac{\mathrm{PVp}}{\pi \mathrm{R}}\right)^{1 / 2} \exp \left[-\frac{\mathrm{P}}{4 \mathrm{RVp}}(\mathrm{R}-\mathrm{Vp})^{2}\right] \\
& -\frac{1}{2}\left(1+\mathrm{P}+\frac{\mathrm{PVp}}{\mathrm{R}}\right) \exp (\mathrm{P}) \operatorname{erfc}\left[\left(\frac{\mathrm{P}}{4 \mathrm{RVp}}\right)^{1 / 2}(\mathrm{R}+\mathrm{Vp})\right]
\end{aligned}
$$

em que o valor de $\mathrm{Vp}_{0}$ é calculado por

$$
\mathrm{Vp}_{0}=\frac{\mathrm{vt}_{0}}{\mathrm{~L}}
$$

\section{Equação de transporte - concentração no fluxo $\left(C_{f}\right)$}

Em muitas situações experimentais é preferível tomar as medidas de concentração no percolado, ao invés da concentração na solução do solo, ou concentração residente. Este é o caso quando se analisam concentrações de solutos em efluentes, obtidas em experimentos com colunas de solo, lisímetros ou poços subfreáticos.

A concentração no fluxo, $\mathrm{C}_{\mathrm{f}}\left(\mathrm{M} \mathrm{L}^{-3}\right)$, é definida como a massa de soluto por unidade de volume do fluido passando através de uma seção transversal durante um intervalo de tempo elementar (Kreft \& Zuber, apud Parker \& van Genuchten, 1984), isto é: 


$$
\mathrm{C}_{\mathrm{f}}=\frac{\mathrm{J}_{\mathrm{s}}}{\mathrm{q}}
$$

Verifica-se, portanto, que concentrações no efluente são concentrações no fluxo.

A relação entre concentração no fluxo e concentração residente é dada pela equação (Parker \& van Genuchten, 1984; van Genuchten \& Wierenga, 1986)

$$
\mathrm{C}_{\mathrm{f}}=\mathrm{C}_{\mathrm{r}}-\frac{\mathrm{D}}{\mathrm{v}} \frac{\partial \mathrm{C}_{\mathrm{r}}}{\partial \mathrm{x}}
$$

A Eq. 3 pode ser escrita para a concentração no fluxo a partir da Eq. 16, obtendo-se:

$$
\mathrm{R} \frac{\partial \mathrm{C}_{\mathrm{f}}}{\partial \mathrm{t}}=\mathrm{D} \frac{\partial^{2} \mathrm{C}_{\mathrm{f}}}{\partial \mathrm{x}^{2}}-\mathrm{v} \frac{\partial \mathrm{C}_{\mathrm{f}}}{\partial \mathrm{x}}
$$

A Eq. 17 é semelhante à Eq. 3, diferindo apenas no fato da concentração residente $\left(\mathrm{C}_{\mathrm{r}}\right)$ estar substituída pela concentração no fluxo $\left(\mathrm{C}_{\mathrm{f}}\right)$.

\section{Condições iniciais e de contorno - concentração no fluxo}

As condições iniciais e de contorno a que a Eq. 17 é submetida em sua solução particular, escritas para $C_{\mathrm{f}}(\mathrm{x}, \mathrm{t})$, são obtidas a partir das equações 5 a 7, utilizando-se a Eq. 16 . Obtém-se, então,

$$
\begin{gathered}
C_{f}(x, 0)=C_{i} \\
C_{f}(0, t)= \begin{cases}C_{0} & 0<t \leq t_{0} \\
0 & t>t_{0}\end{cases} \\
\frac{\partial C_{f}}{\partial x}(\infty, t)=0
\end{gathered}
$$

Observa-se que o modelo de transporte para $\mathrm{C}_{\mathrm{f}}$ difere do modelo para $\mathrm{C}_{\mathrm{r}}$ apenas quanto à condição de contorno de entrada do tipo 3 para $\mathrm{C}_{\mathrm{r}}$ (Eq. 6), a qual é transformada em uma condição do tipo 1, para $\mathrm{C}_{\mathrm{f}}$ (Eq. 19); ambas são dadas para a aplicação de um pulso.

\section{Solução para $\mathrm{C}_{\mathrm{f}}-$ Modelo 2}

A solução da Eq. 17, submetida às condições iniciais e de contorno descritas pelas equações 18 a 20, é (Parker \& van Genuchten, 1984)

$C_{f}(x, t)=\left\{\begin{array}{lc}C_{i}+\left(C_{0}-C_{i}\right) A_{1}(x, t) & 0<t \leq t_{0} \\ C_{i}+\left(C_{0}-C_{i}\right) A_{1}(x, t)-C_{0} A_{1}\left(x, t-t_{0}\right) & t>t_{0}\end{array}\right.$

em que:

$\mathrm{A}_{1}(\mathrm{x}, \mathrm{t})=\frac{1}{2} \operatorname{erfc}\left[\frac{\mathrm{Rx}-\mathrm{vt}}{2(\mathrm{DRt})^{1 / 2}}\right]+\frac{1}{2} \exp \left(\frac{\mathrm{vx}}{\mathrm{D}}\right) \operatorname{erfc}\left[\frac{\mathrm{Rx}+\mathrm{vt}}{2(\mathrm{DRt})^{1 / 2}}\right]$

As equações 23 e 24, obtidas a partir das equações 21 e 22 , respectivamente, para $\mathrm{x}$ igual a $\mathrm{L}$, são a solução para $\mathrm{C}_{\mathrm{f}}$ em termos de Vp e P:

$C_{f}(V p)=\left\{\begin{array}{lc}C_{i}+\left(C_{0}-C_{i}\right) A_{1}(V p) & 0<V p \leq \mathrm{Vp}_{0} \\ C_{i}+\left(C_{0}-C_{i}\right) A_{1}(V p)-C_{0} A_{1}\left(V p-V p_{0}\right) & V p>V p_{0}\end{array}\right.$ em que:

$\mathrm{A}_{1}(\mathrm{Vp})=\frac{1}{2} \operatorname{erfc}\left[\left(\frac{\mathrm{P}}{4 \mathrm{RVp}}\right)^{1 / 2}(\mathrm{R}-\mathrm{Vp})\right]+\frac{1}{2} \exp (\mathrm{P}) \operatorname{erfc}\left[\left(\frac{\mathrm{P}}{4 \mathrm{RVp}}\right)^{1 / 2}(\mathrm{R}+\mathrm{Vp})\right]$

\section{Outros modelos de transporte}

Dois outros modelos também foram implementados no programa; no primeiro, a difusão é negligenciada e, no segundo modelo, além da difusão ser negligenciada, o fator de retardamento $(\mathrm{R})$ será considerado igual a 1 .

\section{Modelo negligenciando a difusão - Modelo 3}

Este modelo emprega as equações

$$
C(x, t)=\left\{\begin{array}{lc}
C_{i}+\left(C_{0}-C_{i}\right) A_{0}(x, t) & 0<t \leq t_{0} \\
C_{i}+\left(C_{0}-C_{i}\right) A_{0}(x, t)-C_{0} A_{0}\left(t-t_{0}\right) & t>t_{0}
\end{array}\right.
$$

em que:

$$
A_{0}(x, t)=\frac{1}{2} \operatorname{erfc}\left[\frac{R x-v t}{2(D R t)^{1 / 2}}\right]
$$

ou:

$$
C(V p)=\left\{\begin{array}{lr}
C_{i}+\left(C_{0}-C_{i}\right) A_{0}(V p) & 0<V p \leq V p_{0} \\
C_{i}+\left(C_{0}-C_{i}\right) A_{0}(V p)-C_{0} A_{0}\left(V p-V p_{0}\right) & V p>V p_{0}
\end{array}\right.
$$

em que:

$$
\mathrm{A}_{0}(\mathrm{Vp})=\frac{1}{2} \operatorname{erfc}\left[\left(\frac{\mathrm{P}}{4 \mathrm{RVp}}\right)^{1 / 2}(\mathrm{R}-\mathrm{Vp})\right]
$$

em que $\mathrm{C}$ é a concentração do soluto no efluente, $\mathrm{M} \mathrm{L}^{-3}$.

Os resultados obtidos com o emprego da Eq. 27 aproximam-se daqueles obtidos com as Eqs. 12 e 23, para valores de $\mathrm{P}$ relativamente grandes (van Genuchten \& Wierenga, 1986). Observa-se que o segundo membro da Eq. 28 é o primeiro termo do segundo membro das Eqs. 13 e 24. Ainda que este modelo não seja formalmente aplicável a experimentos de laboratório ou campo, sua forma simples e o fato dos resultados se aproximarem aos obtidos com as soluções analíticas, quando P é grande, fazem com que ele possa ser considerado uma ferramenta atraente, por gerar expressões simples e aproximadas para D em termos dos parâmetros medidos.

\section{Modelo negligenciando a difusão e para $\mathbf{R}$ igual a 1 - Modelo 4}

A equação de transporte de soluto para este modelo é

$$
\frac{\partial \mathrm{C}}{\partial \mathrm{t}}=\mathrm{E} \frac{\partial^{2} \mathrm{C}}{\partial \mathrm{x}^{2}}
$$

em que E é um coeficiente de dispersão mecânica, que não deve ser confundido com o coeficiente de difusão, da equação de Fick, pelo fato de E ser independente da concentração do soluto.

A solução da Eq. 29 para a aplicação de um pulso de soluto, é (Kirkham \& Powers, 1972; Ferreira, 2001) 


$$
\frac{\mathrm{C}(\mathrm{x}, \mathrm{t})}{\mathrm{C}_{0}}=\frac{1}{2}\left\{\left[\operatorname{erf} \frac{\mathrm{x}+\mathrm{x}_{0}-\mathrm{vt}}{2(\mathrm{Et})^{1 / 2}}\right]-\left[\operatorname{erf} \frac{\mathrm{x}-\mathrm{vt}}{2(\mathrm{Et})^{1 / 2}}\right]\right\}
$$

ou

$$
\frac{\mathrm{C}(\mathrm{Vp})}{\mathrm{C}_{0}}=\frac{1}{2}\left\{\left[\operatorname{erf} \frac{1+\frac{\mathrm{x}_{0}}{\mathrm{~L}}-\mathrm{Vp}}{2[\mathrm{E} \mathrm{Vp} /(\mathrm{vL})]^{1 / 2}}\right]-\left[\operatorname{erf} \frac{1-\mathrm{Vp}}{2[\mathrm{EVp} /(\mathrm{vL})]^{1 / 2}}\right]\right\}
$$

em que o valor de $\mathrm{x}_{0}(\mathrm{~L})$ pode ser obtido pela relação:

$$
\mathrm{x}_{0}=\frac{\mathrm{Vs}}{\alpha \mathrm{A}}
$$

em que:

$$
\begin{aligned}
& \text { Vs - volume da solução, } \mathrm{L}^{3} \\
& \mathrm{~A} \text { - área transversal, } \mathrm{L}^{2} \\
& \alpha \text { - porosidade efetiva, } \mathrm{L}^{3} \mathrm{~L}^{-3}
\end{aligned}
$$

\section{Balanço de massa}

O balanço de massa refere-se ao cálculo das quantidades de soluto aplicada, retida e efluente. Pode estar relacionado tanto a determinada profundidade em um perfil de solo, quanto a uma coluna de solo.

$$
\begin{gathered}
\mathrm{Q}_{1}=\mathrm{vC}_{0} \mathrm{t}=\mathrm{R} \int_{0}^{\infty}\left[\mathrm{C}_{\mathrm{r}}(\mathrm{x}, \mathrm{t})-\mathrm{C}_{\mathrm{i}}\right] \mathrm{dx} \\
\mathrm{Q}_{2}=\mathrm{v} \int_{0}^{\mathrm{t}}\left[\mathrm{C}_{0}-\mathrm{C}_{\mathrm{f}}(\mathrm{L}, \tau)\right] \mathrm{d} \tau=\mathrm{R} \int_{0}^{\mathrm{L}}\left[\mathrm{C}_{\mathrm{r}}(\mathrm{x}, \mathrm{t})-\mathrm{C}_{\mathrm{i}}\right] \mathrm{dx} \\
\mathrm{Q}_{3}=\mathrm{Q}_{1}-\mathrm{Q}_{2}
\end{gathered}
$$

em que:

$\mathrm{Q}_{1}$ - quantidade de soluto aplicada por unidade de área, $\mathrm{M} \mathrm{L}^{-2}$

$\mathrm{Q}_{2}$ - quantidade de soluto por unidade de área, localizada até a profundidade L, no perfil do solo, a qual também pode representar o comprimento de uma coluna de solo, $\mathrm{M} \mathrm{L}^{-2}$

$\mathrm{Q}_{3}$ - quantidade de soluto por unidade de área, percolada abaixo de dada profundidade L, $\mathrm{M} \mathrm{L}^{-2}$

$\mathrm{Na}$ Eq. 34 declara-se que a quantidade de soluto aplicada menos a quantidade que deixa a coluna (segundo membro), deve estar armazenada no perfil de solo, até a profundidade L, ou na coluna de solo de comprimento L (terceiro membro).

\section{Desenvolvimento do programa computacional}

O programa computacional, denominado Disp, foi desenvolvido em Delphi (Borland Software Corporation). O desenvolvimento compreendeu a implementação de procedimentos relativos ao cálculo das funções erro e erro complementar, cálculo do produto $\exp (\mathrm{P})$ por erfc(y), método de ajuste de curvas, balanço de massa e o desenvolvimento da interface. Os procedimentos são descritos a seguir.

a) Cálculo das funções erro e erro complementar

A função erro (erf) e a função erro complementar (erfc) são calculadas, respectivamente, pelas equações

$$
\begin{gathered}
\operatorname{erf}(y)=\frac{2}{\sqrt{\pi}} \int_{0}^{y} \exp \left(-w^{2}\right) d w \\
\operatorname{erfc}(y)=1-\operatorname{erf}(y)
\end{gathered}
$$

em que y é o argumento da função erro e w é uma variável auxiliar de integração.

Foram implementados dois métodos numéricos para o cálculo da integral da Eq. 36, podendo o usuário optar por um deles. O primeiro método foi Quadratura de Gauss (Gerald \& Wheatleay, 1984) com quatro termos, cujo ajuste é de sétimo grau; o outro método é o do Polinômio P5 de Newton-Cotes, com precisão de aproximação de sétima ordem.

Os cálculos de erfc(y), tanto com o método de integração de Quadratura de Gauss, quanto com o Polinômio P5 de Newton-Cotes, serão aplicados quando o valor do argumento da função erro complementar estiver entre $-5,9$ e 5,9, isto é, $-5,9 \leq \mathrm{y} \leq 5,9$. Para valores de y inferiores a $-5,9$, adotou-se erfc(y) igual a 2; para valores de y maiores que 5,9, adotou-se erfc(y) igual a 0. Como o módulo do argumento de erfc(y), nos modelos 1,2 e 3 , cresce principalmente com $P$, e se considerando que, quanto maior $\mathrm{P}$, mais próximos serão os resultados obtidos com esses modelos, espera-se que o procedimento de truncamento não acarrete imprecisões significativas nas estimativas dos parâmetros P e R. Geralmente, o truncamento será necessário para valores de $\mathrm{P}$ a partir de 30 .

b) Cálculo do produto $\exp (\mathrm{P}) \operatorname{erfc}(\mathrm{y})$

Existem algumas dificuldades em se conseguir a precisão necessária no cálculo do produto $\exp (\mathrm{P})$ por $\operatorname{erfc}(\mathrm{y})$, presente nos modelos 1 e 2 , especialmente quando o valor de $\mathrm{P}$ é grande e, conseqüentemente, erfc(y) é pequeno. Por exemplo, com $\mathrm{P}=30, \mathrm{R}=1$, e $\mathrm{Vp}=1$, tem-se $\exp (\mathrm{P})=7,07 * 10^{13}$ e $y=7,5$, que supera o limite superior de truncamento para y, na função erfc. Toride et al. (1999) relatam procedimentos originários de Abramowitz \& Stegun (1972), implementados no CXTFIT, para execução do cálculo do produto de $\exp (\mathrm{P})$ por erfc(y). No Disp, alguns dos procedimentos de Abramowitz \& Stegun (1972) foram adaptados a uma função denominada prod(P,y), empregando-se valores modificados dos extremos de y. Esta função é descrita a seguir.

Caso y $>5,6$

$\operatorname{prod}(\mathrm{P}, \mathrm{y})=\frac{1}{\sqrt{\pi}} \frac{\exp \left(\mathrm{P}-\mathrm{y}^{2}\right)}{(\mathrm{y}+0,5 /(\mathrm{y}+1 /(\mathrm{y}+1,5 /(\mathrm{y}+2 /(\mathrm{y}+2,5 /(\mathrm{y}+1))))))}$

Caso $\mathrm{y}<0$

$$
\operatorname{prod}(\mathrm{P}, \mathrm{y}))=2 \exp (\mathrm{P})-\operatorname{prod}(\mathrm{P},-\mathrm{y})
$$

Caso $(P>170$ e $y \leq 0)$ ou $\left(\left|P-y^{2}\right|>170\right.$ e $\left.y>0\right), \operatorname{prod}(P, y)=0$.

Outros casos $\operatorname{prod}(\mathrm{P}, \mathrm{y})=\exp (\mathrm{P}) \operatorname{erfc}(\mathrm{y})$

\section{c) Método de ajuste de curvas}

Para os modelos 1, 2 e 3, utilizou-se um método de mínimos quadrados não-linear para ajuste de curvas, ou seja, para obtenção dos estimadores dos parâmetros $\mathrm{P}$ e R. Este método consiste em se adotar os estimadores que minimizam a soma 
dos quadrados dos desvios (Rs), entre os valores estimados e observados de concentração em relação ao número de volume de poros. O método de mínimos quadrados não-linear utilizado foi o de Levenberg-Marquardt (Ratkowsky, 1989; Seber \& Wild, 1989; Cunha, 1993). Este método requer a obtenção de derivadas parciais para a curva de concentração, em relação aos parâmetros $\mathrm{P}$ e $\mathrm{R}$, em cada ponto da curva em que se tenha uma observação experimental. Essas derivadas foram calculadas numericamente empregando-se o polinômio de diferenças progressivas de Newton de ordem 4.

Aliado ao método de Levenberg-Marquardt, implementouse uma rotina para verificar se na vizinhança dos valores dos parâmetros $\mathrm{P}$ e R, obtidos com este método, existem valores que proporcionem melhor ajuste. Tal procedimento mostrouse necessário para evitar possíveis erros decorrentes dos critérios de convergência e dos métodos numéricos de diferenciação utilizados no método de mínimos quadrados.

Os valores iniciais de $\mathrm{P}$ e $\mathrm{R}$ necessários ao processamento do método de mínimos quadrados, podem ser fornecidos pelo usuário; entretanto, como procedimento padrão, o programa utiliza valores iniciais padrões $(\mathrm{P}=25$ e $\mathrm{R}=0,9)$; os referidos valores serão, então, utilizados para obtenção de valores ajustados de $\mathrm{P}$ e R para o Modelo 3, os quais serão os valores iniciais empregados para se obter valores ajustados de P e R para o modelo (1 ou 2) com o qual se esteja trabalhando.

Foi implementado, também, um método que processa uma superfície de resposta $\mathrm{Rs}(\mathrm{P}, \mathrm{R})$, ou seja, calcula valores da soma de quadrados dos desvios para cada combinação de $\mathrm{P}$ e R, indicando qual combinação desses parâmetros resulta em menor Rs. O gráfico da superfície de resposta permite observar a convergência para o mínimo da soma de quadrados dos desvios. Neste método, valores iniciais e finais de $\mathrm{P}$ e R, bem como incrementos em $\mathrm{P}$ e R, devem ser fornecidos.

Para o modelo 4, que requer o ajustamento de apenas um parâmetro (E), adotou-se um simples procedimento de busca. Devem ser fornecidos valores iniciais, finais e incrementais de E. Para cada valor de E avaliado no procedimento de busca, é feito o cálculo de Rs. O programa indica, então, qual valor de E proporciona o menor Rs.

\section{d) Balanço de massa}

O cálculo das integrais presentes na Eq. 34 foi implementado através do método numérico Regra 1/3 de Simpson, com extrapolação de Richardson (Gerald \& Wheatley, 1984; Cunha, 1993).

\section{Testes para validação}

O programa CXTFIT tem sido documentado e utilizado há vários anos (Parker \& van Genuchten, 1984; Toride et al., 1999). A validação do programa Disp foi verificada por meio de testes comparativos entre os resultados obtidos com este programa e aqueles obtidos com o CXTFIT, relativos ao cálculo dos parâmetros P e R, com os modelos 1, 2 e 3, cuja comparação consistirá no cálculo da diferença percentual, conforme a equação

$$
\text { DPer }=100 \frac{\operatorname{VPar}_{\text {CXTFIT }}-\operatorname{VPar}_{\text {DISP }}}{\operatorname{VPar}_{\text {CXTFIT }}}
$$

em que:
DPer - diferença percentual entre os parâmetros P ou $\mathrm{R}$, calculados com o Disp e CXTFIT, \%

VPar - valor do parâmetro P ou R, calculado com o Disp e CXTFIT

Utilizaram-se dados de experimentos em coluna de solo. Dados de quatro desses experimentos foram obtidos da literatura (van Genuchten \& Wierenga, 1986). Foram considerados, também, dados de três experimentos realizados em laboratórios dos Departamentos de Engenharia Agrícola e de Solos da Universidade Federal de Viçosa, dois dos quais referentes ao estudo de Oliveira et al. (2004), no qual o Disp foi empregado.

No Disp, os resultados obtidos com o método de mínimos quadrados não-linear de Levenberg-Marquardt (procedimento padrão do programa) foram também comparados com os obtidos por meio da análise da superfície de resposta $\mathrm{Rs}(\mathrm{P}, \mathrm{R})$ (procedimento alternativo).

\section{RESULTADOS E DISCUSSÃO}

\section{Programa desenvolvido}

O programa desenvolvido (Disp) possui uma interface gráfica que facilita os procedimentos de entrada de dados. É provido de um sistema de ajuda que orienta quanto ao uso do programa e apresenta, detalhadamente, a metodologia empregada em seu desenvolvimento. Os dados de entrada podem ser salvos em arquivos binários e incluir a identificação e os comentários relativos ao modelo físico experimental. Na Figura 1 apresenta-se o formulário inicial no qual são procedidas a entrada de dados e a apresentação dos valores obtidos de P, D (ou E) e R, após os cálculos. O formulário é provido de uma calculadora para função erro e função erro complementar, localizada no canto superior direito; abaixo desta calculadora se encontra o campo para escolha do modelo a ser empregado. O Modelo 4 é o único que não consta neste campo e é disponibilizado, como opção, quando os dados de entrada são referentes à aplicação de um pulso.

Os dados de saída são apresentados nos formulários do programa, podendo ser registrados em arquivos de

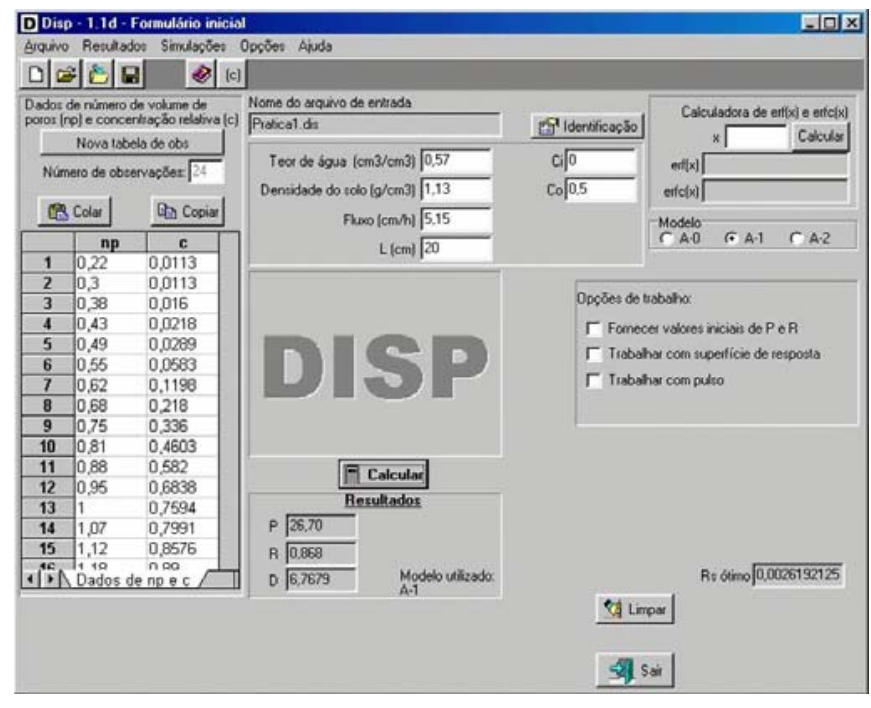

Figura 1. Formulário inicial do programa 
resultados, no formato texto. Os gráficos podem ser salvos ou exportados para outros programas. Os formulários de resultados são acessados com o menu existente no formulário inicial.

A Figura 2 apresenta um formulário contendo gráfico e planilha com valores observados e calculados de concentração relativa (c), para a curva de efluente, em função do número de volume de poros. Neste formulário é possível se obter curvas com outros valores de $\mathrm{P}$ e R, permitindo observar-se os efeitos da variação desses parâmetros sobre a concentração relativa.

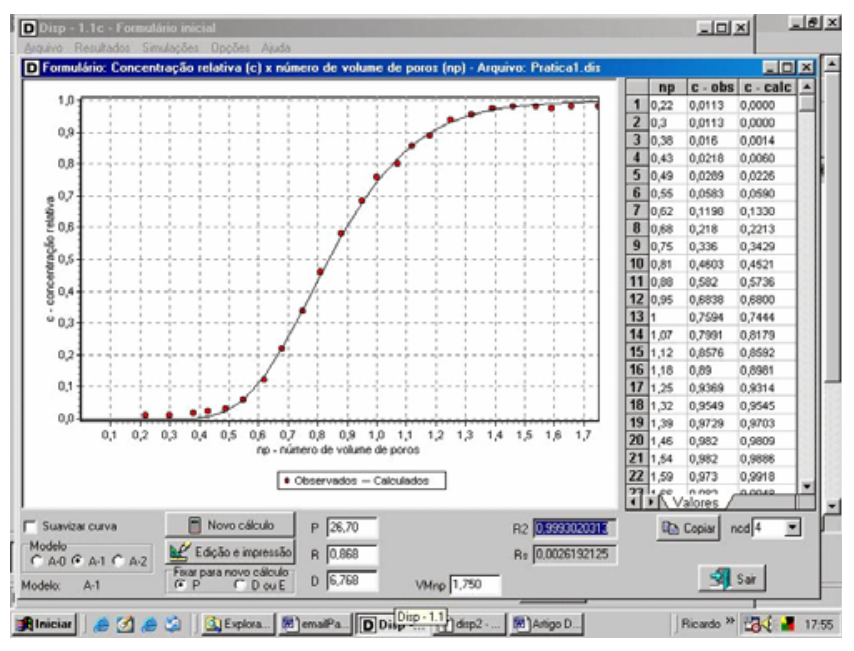

Figura 2. Formulário de resultados apresentando valores observados e calculados de concentração relativa, referentes à curva de efluente, em função do número de volume de poros

Vê-se, na Figura 3, o formulário no qual o movimento de solutos no perfil do solo e o balanço de massa podem ser simulados, considerando-se diferentes valores das variáveis de entrada e parâmetros dos modelos de transporte. São gerados gráficos e tabelas mostrando a variação espacial e temporal de concentrações no perfil do solo.

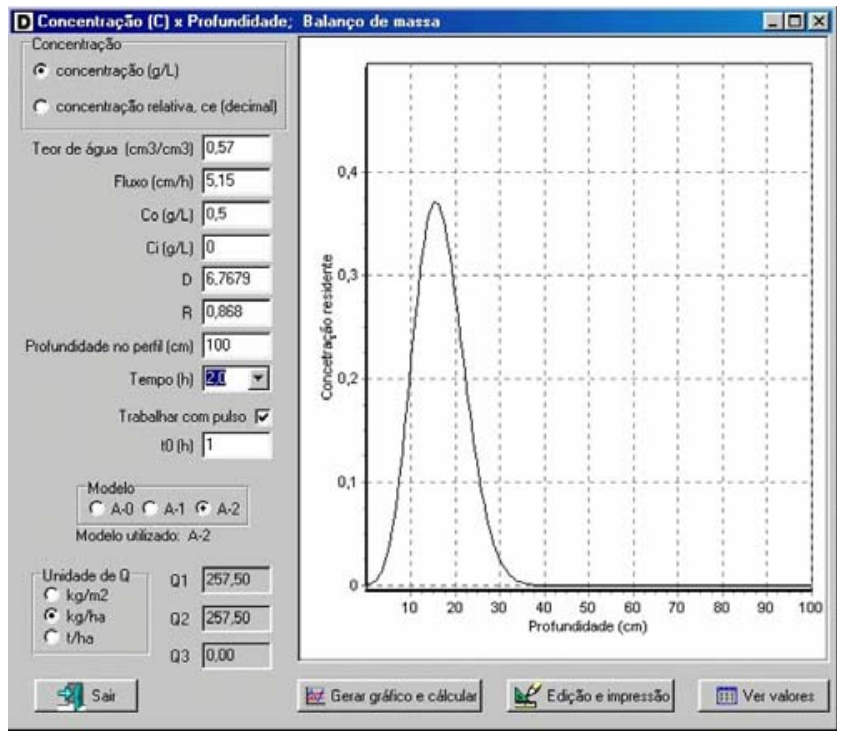

Figura 3. Variação espacial e temporal da concentração no perfil do solo

\section{Testes para validação}

Nas Tabelas 1 e 2 apresentam-se os resultados das comparações entre os resultados dos cálculos de $\mathrm{P}$ e R, obtidos com o Disp e o CXTFIT, para 7 experimentos.

Conforme se observa nas Tabelas 1 e 2, obtiveram-se resultados muito próximos, para $\mathrm{P}$ e R, indicando a equivalência dos programas Disp e CXTFIT na obtenção desses parâmetros de transporte. As diferenças percentuais máxima e mínima, DPer, foram 0,39 e $-0,40 \%$. Diferenças percentuais desta ordem implicam em que não haveria diferença visual em curvas de efluente ou curvas de distribuição da concentração de solutos no perfil do solo, obtidas com os dois programas. Para o fator de retardamento, algumas das diferenças percentuais ocorreram unicamente em virtude do número de casas decimais fornecido no resultado; o Disp fornece três casas decimais e o CXTFIT quatro.

Nos resultados da Tabela 1 não se observa tendência

Tabela 1. Valores dos parâmetros número de Peclet (P) e fator de retardamento (R), calculados com os programas Disp e CXTFIT e diferença percentual (DPer)

\begin{tabular}{|c|c|c|c|c|c|c|c|c|c|c|c|c|}
\hline \multirow{2}{*}{$N^{0}$ Exp. } & \multirow{2}{*}{ Soluto } & \multirow{2}{*}{$\mathrm{N}^{0}$ Obs. } & & \multicolumn{3}{|c|}{ Modelo 1} & \multicolumn{3}{|c|}{ Modelo 2} & \multicolumn{3}{|c|}{ Modelo 3} \\
\hline & & & & Disp & CXTFIT & DPer & Disp & CXTFIT & DPer & Disp & CXTFIT & DPer \\
\hline \multirow[t]{2}{*}{1} & ${ }^{3} \mathrm{H}_{2} \mathrm{O}$ & 20 & $\mathrm{P}$ & 29,53 & 29,54 & 0,03 & 30,00 & 30,00 & 0,00 & 30,49 & 30,49 & 0,00 \\
\hline & & & $\mathrm{R}$ & 0,967 & 0,967 & 0,00 & 1,000 & 1,000 & 0,00 & 0,968 & 0,968 & 0,00 \\
\hline \multirow[t]{2}{*}{2} & $\mathrm{Cr}^{6+}$ & 15 & $P$ & 19,17 & 19,19 & 0,10 & 19,62 & 19,65 & 0,15 & 20,10 & 20,11 & 0,05 \\
\hline & & & $\mathrm{R}$ & 1,281 & 1,280 & $-0,08$ & 1,348 & 1,349 & 0,07 & 1,284 & 1,284 & 0,00 \\
\hline \multirow[t]{2}{*}{3} & $\mathrm{Cl}^{-}$ & 29 & $\mathrm{P}$ & 253,55 & 253,10 & $-0,18$ & 252,93 & 253,6 & 0,26 & 254,50 & 254,10 & $-0,16$ \\
\hline & & & $\mathrm{R}$ & 0,918 & 0,918 & 0,00 & 0,921 & 0,921 & 0,00 & 0,918 & 0,918 & 0,00 \\
\hline \multirow[t]{2}{*}{4} & ${ }^{3} \mathrm{H}_{2} \mathrm{O}$ & 10 & $\mathrm{P}$ & 26,32 & 26,31 & $-0,04$ & 26,72 & 26,76 & 0,15 & 27,26 & 27,26 & 0,00 \\
\hline & & & $\mathrm{R}$ & 0,937 & 0,937 & 0,00 & 0,973 & 0,973 & 0,00 & 0,938 & 0,938 & 0,00 \\
\hline \multirow[t]{2}{*}{5} & Amônio & 8 & $P$ & 6,57 & 6,574 & 0,06 & 7,04 & 7,027 & $-0,19$ & - & - & - \\
\hline & & & $\mathrm{R}$ & 1,921 & 1,9201 & $-0,05$ & 2,220 & 2,2195 & $-0,02$ & - & - & - \\
\hline \multirow[t]{2}{*}{6} & Fosfato & 14 & $P$ & 1,35 & 1,347 & $-0,22$ & 1,24 & 1,236 & $-0,32$ & - & - & - \\
\hline & & & $\mathrm{R}$ & 4,306 & 4,3065 & 0,01 & 8,791 & 8,8021 & 0,13 & - & - & - \\
\hline \multirow[t]{2}{*}{7} & $\mathrm{Cl}^{-}$ & 24 & $\mathrm{P}$ & 26,24 & 26,34 & 0,36 & 26,70 & 26,81 & 0,39 & - & - & - \\
\hline & & & $\mathrm{R}$ & 0,836 & 0,8327 & $-0,40$ & 0,868 & 0,8646 & $-0,39$ & - & - & - \\
\hline
\end{tabular}

* Fonte: Experimentos 1 a 4 - van Genuchten \& Wierenga (1986); experimentos 5 e 6 - Departamento de Solos, UFV; experimento 7 - Departamento de Engenharia Agrícola, UFV 
Tabela 2. Médias dos módulos, valores máximos e valores mínimos da diferença percentual (DPer)

\begin{tabular}{lrccc}
\hline \multirow{2}{*}{ Estatisticas } & & \multicolumn{3}{c}{ Modelo } \\
\cline { 3 - 5 } Média do módulo de DPer (\%) & $\mathrm{P}$ & $\mathbf{1}$ & $\mathbf{2}$ & $\mathbf{3}$ \\
& $\mathrm{R}$ & 0,08 & 0,21 & 0,05 \\
Máxima DPer (\%) & $\mathrm{P}$ & 0,36 & 0,09 & 0,00 \\
& $\mathrm{R}$ & 0,01 & 0,13 & 0,05 \\
Mínima DPer (\%) & $\mathrm{P}$ & $-0,22$ & $-0,32$ & $-0,00$ \\
& $\mathrm{R}$ & $-0,40$ & $-0,39$ & 0,00 \\
\hline
\end{tabular}

geral de superestimativa ou subestimativa, nos valores dos parâmetros obtidos com o Disp, em relação àqueles obtidos com o CXTFIT (os termos superestimativa e subestimativa são aqui utilizados para facilitar a discussão, apesar de não condizerem com as reduzidas diferenças percentuais obtidas), nem se observou tendência de superestimativa ou subestimativa, conforme o modelo considerado.

Nota-se expressiva amplitude do intervalo dos valores dos parâmetros obtidos com os programas, apresentados na Tabela 1. O número de Peclet variou de 1,24 (amônio, Modelo 2) a 254,5 ( $\mathrm{Cl}^{-}$, Modelo 3), enquanto o fator de retardamento oscilou de 0,836 ( $\mathrm{Cl}^{-}$, Modelo 1) a 8,8 (fosfato, Modelo 2). Nesses intervalos os dois programas foram equivalentes quanto às estimativas de $\mathrm{P} e$ $\mathrm{R}$, independente da magnitude desses parâmetros.

Não ocorreram diferenças entre os resultados do Disp obtidos com o método de mínimos quadrados não-linear (Levenberg-Marquardt) e com a ferramenta para geração da superfície de resposta Rs(P,R), ou seja, da soma dos quadrados dos desvios em função do número de Peclet e do fator de retardamento. Este resultado era previsto, considerando que em ambos os métodos são utilizados os mesmos procedimentos relativos à função erro complementar e ao cálculo do produto de $\exp (\mathrm{P})$ por erfc(y). Um exemplo da superfície de resposta obtida com o Disp é apresentado na Figura 4, referente ao experimento número 7 , Modelo 2, listado na Tabela 1 .

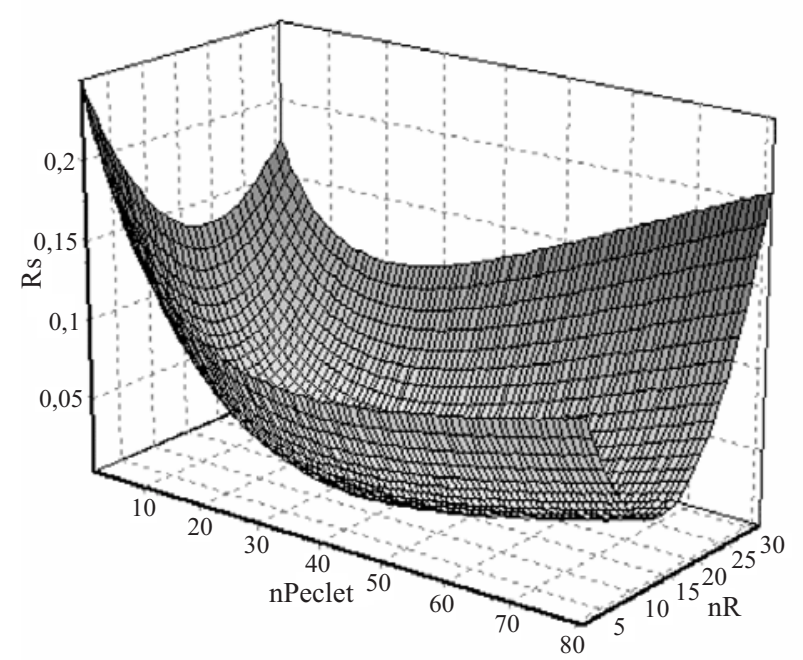

Figura 4. Superfície de resposta gerada no Disp para a soma dos quadrados dos desvios em função do número de Peclet e do fator de retardamento (nos eixos rotulados 'nPeclet' $\mathrm{e}$ 'nR' estão os índices ou ordem dos valores do número de Peclet e do fator de retardamento avaliados)

\section{CONCLUSÕES}

1. Em testes comparativos quanto aos cálculos dos parâmetros número de Peclet $(\mathrm{P})$ e fator de retardamento $(\mathrm{R})$, processados com o programa desenvolvido, Disp, e o programa CXTFIT, não se obtiveram diferenças percentuais superiores a $0,4 \%$, em módulo, indicando a equivalência dos dois programas na obtenção desses parâmetros.

2. A interface gráfica do programa Disp possibilita simplicidade quanto aos procedimentos de entrada de dados e acesso aos resultados, facilitando sua utilização na obtenção de parâmetros de transporte de solutos no solo, a partir das curvas de efluente ou para simular, durante um processo de deslocamento de fluidos miscíveis, a variação espacial e temporal da concentração de solutos no perfil do solo e o balanço de massa a determinada profundidade no perfil.

\section{LITERATURA CITADA}

Abramowitz, M.; Stegun, I. A. Handbook of mathematical functions with formulas, graphs, and mathematical tables. 10.ed. Washington: United States Government Printing Office, 1972. $1046 \mathrm{p}$.

Cunha, C. Métodos numéricos para as engenharias e ciências aplicadas. Campinas: UNICAMP, 1993. 265p.

Ferreira, P. A. Drenagem. In: Curso de Engenharia de Irrigação. Módulo XI. Brasília: ABEA Superior. 2001. 111p.

Gerald, C. F.; Wheatley, P. O. Applied numerical analysis. San Luis Obispo: Addison-Wesley Publishing Company, 1984. 579p.

Kirkham, D.; Powers, W. L. Advanced soil physics. New York: John Wiley \& Sons, 1972. 534p.

Oliveira, E. M. M.; Ruiz, H. A; Ferreira, P. A.; Alvarez V., V. H.; Borges Júnior, J. C. F. Fatores de retardamento e coeficientes de dispersão-difusão de fosfato, potássio e amônio em solos de Minas Gerais. Revista Brasileira de Engenharia Agrícola e Ambiental, Campina Grande, v.8, n.2-3, p.196-203, 2004.

Parker, J. C.; van Genuchten, M. T. Determining transport parameters from laboratory and field tracer experiments. Virginia Agricultural Experiment Station. Blacksbug: Virginia Polytechnic Institute and State University.1984. 89p. Bulletin 87-3

Ratkowsky, D. A. Handbook of nonlinear regression models. New York: Marcel Dekker, 1989. 241p.

Reichardt, K. Dinâmica da matéria e da energia em ecossistemas. 2ed., Piracicaba: ESALQ/ USP, 1996, 505p.

Seber, G. A. F.; Wild, C. J. Nonlinear regression. New York : John Wiley, 1989. 768p.

Toride, N.; Leij, F. J.; van Genuchten, M. T.; The CXTFIT code for estimating transport parameters from laboratory or field tracer experiments. Version 2.1. Research report No. 137. Riverside, United States Salinity Laboratory, Agricultural Research Service, United States, Department of Agriculture, 1999. 119p. van Genuchten, M. T.; Wierenga, P. J. Solute dispersion coefficients and retardation factors. In: Methods of soil analysis, Part 1. Madison: American Society of Agronomy - Soil Science Society of America, 1986. p.1025-1054. 